\title{
How Participation at Different Hierarchical Levels Can Have an Impact on the Design and Implementation of Health Information Systems at the Grass Root Level - A Case Study from India*
}

\author{
Zubeeda Banu Quraishy \\ Department of Informatics, University of Oslo \\ Gaustadalleen 23, PO. Box. 080 Blindern, NO - 0316 Oslo, Norway \\ Mailing Address: G-3, M.I.G Flats, Fore-Shore Estate, Chennai-600 028, India \\ Tel.: 98400 20114; Fax: + 91-44-42104 106 \\ zubeedaq@ifi.uio.no
}

\begin{abstract}
To build the effective information systems Scandinavian tradition emphasizes and encourages the participatory design methods which involves both designers and users equally in building the systems. While the main intention of Scandinavian tradition is to refocus the dominant orientation of systems developers by involving users but one really wonders whether it can be applied in the context of developing countries particularly in a country like India where the structures are rigid, hierarchical and strongly bureaucratic in nature. In spite of the fact that health sector being a key social area in India's developmental administration it is highly fragmented and receives low priority. In India, while the general administration is mostly concentrated at the district level, health and family welfare administration has been centralized at state and central levels. This coupled with the excessive concentration of powers in the secretariat system of governance, has considerably centralized planning and monitoring. Keeping in view the above complexities and challenging conditions this paper tries to understand using case study as a method how participation at different levels affects the design and implementation of Health Information Systems in primary health care sector in India.
\end{abstract}

Keywords: Participatory Design, Health Information Systems Programme, Primary Health Centres, Users, Developing Countries.

\section{Introduction}

In developed countries where participation is welcomed and encouraged if not in all sectors at least in social sectors like education and health in developing countries in all contexts it is highly discouraged. In developing countries like India there was no tradition of participatory planning and decision making until December 1992 when

* The empirical material for the above case study was collected during my association with HISP project as Project Coordinator, HISP India from January 2001 to April 2005. 
$72^{\text {nd }}$ (Panchayats) and 73rd (Municipalities) constitutional amendment bills, were passed by both the houses of parliament and came in to force in 1993. The above legislations brought in to effect the much talked about decentralisation with a view towards promoting participation and improving the controlling function held by the lower levels of the political system and the communities as it was believed that decentralisation would bring government and its administration closer to the people. Also it was considered that the existence of local political arenas makes it easier for ordinary citizens to participate and exert influence. Thus the legitimacy of public bodies may be considerably boosted by the greater involvement and influence of ordinary people due to the greater 'proximity to politics' that decentralisation entails [2] as this tends to strengthen the state's capacity for implementation. Keeping this in view, the policies were framed in India to improve the involvement of both the decision-makers and the communities at the local level hoping that change occurred at the grass root level and in organisations will not only encourage users to participate in discussions and decisions, but will focus on the issues they identify as important.

Furthermore, it was believed that a decentralised bureaucracy will not only adapt more easily to local identities and norms, which may increase effectiveness but decision-makers who are rooted in the locality and have knowledge of conditions on the ground are better placed to formulate concrete policies for that area than functionaries from the centre [3].

Thus, in order to bring about the desired social change it was considered necessary to have the constitutional support and legislative measures but soon it became clear that they are not the sufficient condition to achieve that goal as social and political structures present in India are highly rigid, hierarchical and strongly bureaucratic in nature.

With this as a back ground the present paper using case study as a method tries to highlight how implementation of Health Information Systems Programme (HISP) with the main objective of strengthening information practices within the Primary Health Care (PHC) sector with the larger aim to improve processes concerning health care delivery for the rural community failed in getting stabilized and sustainable even after five years of implementation efforts as it was thwarted by many challenges mainly due to the lack of participation not only by users but by policy makers and other officials at various levels.

Health Information Systems Programme is an ongoing action research project related to the implementation of information systems for improved local control and use of information at district and sub-district levels in the health sector in Andhra Pradesh.

\section{Situation Analysis}

The Primary health care sector in Andhra Pradesh which is the main interface between the community and the health sector is fragmented and there is no cohesion between the various health departments for whom enormous amount of data is collected at the grass root level by the health worker. While the main duty of the health worker is to perform out reach services to the communities under her purview such as providing Ante Natal Care(ANC), performing institutional deliveries, giving 
immunization to infants and children, providing treatment on minor ailments, and motivating eligible couples for adopting spacing methods and sterlisations, conducting sputum tests of suspected TB patients, reporting and providing immediate care to patients on outbreak of epidemics etc but instead of performing outreach services the health worker is now burdened with achieving targets on performance indicators mainly sterlisations, immunizations, ANC, Post Natal Care and institutional deliveries etc. On $28^{\text {th }}$ of every month all the health workers assemble in primary health centres to collate data collected on the above performance indicators into different registers. Generally the health workers enters the data after performing services into her field diary and then from her field diary the data is aggregated and entered in to the comprehensive register and then from the register the aggregated data is again entered in to different reports like CNA(Community Needs Assessment) report, school health and hostel reports, immunization, Eligible Couple report, Blindness control, Leprosy, Iodine deficiency, malaria and TB programme reports etc. It is quite tedious work and the health workers who are now used to this monotonous work cull out the figures from the registers and enter into reports to be sent to the various officers in charge for the different vertical programmes thus resulting in routine collection, collation, duplication and manipulation of data.

The data collected by the health worker at the cutting edge level is sent vertically upwards with little or no feed back and support from the top to the health worker in the field who needed the information most. In spite of the fact that community use, feedback and feed up is essential for a successful sustainable information system particularly in cases of sensitive issues such as Reproductive Tract Infection(RTI), Sexually Transmitted Infections(STI), HIV/AIDS cases and epidemics like Gastroenteritis(GE) and diarrhea it never happened in the health sector of Andhra Pradesh. It is at this local level that the information is available and where immediate action needs to be taken. On contrary, the use of information is minimally used at the top. Communities and service providers need to share relevant information on the local situation to develop and prioritize strategies together to improve the health situation. Sharing respectful dialogue and resources between communities and service providers can produce positive and lasting improvements by creating a shared vision, goal and objective through approaches that foster equity and shared responsibility.

The transition to an information system that is, an integral part of local governance and part of a social process for development is not only a question of changing of structures and technologies but it challenges deeply rooted values and perceptions with regard to society, technology and health care.

The quality of existing information management practices within the Primary Health Care sector in Andhra Pradesh is extremely poor as the data collected is of poor quality, redundant, aggregated at every level and by the time it reaches the top the situation in the peripheral areas is completely masked. The health workers are under tremendous pressure for data collection and transmission to the top where it is used minimally. Also, major portion of the time of health workers gets wasted in duplicating the data as they have to report almost the same data to various departments using approximately forty forms thus leading to poor quality and fragmentation of the data. The above work is done at the cost of neglecting of providing essential services to the community which is the prime duty of the grass root health worker. 
Except for making the field staff attend routine camps to train them how to motivate eligible couples for sterilizations there were no attempts at all to equip them with additional technological skills, develop a culture of use of information, enhance their capacity in decision making at the PHC level. The structure is so bureaucratic and hierarchical that there is no way of participation of health workers even at the PHC level while participation in the decision making at the higher level is almost a dream. Decentralization which was so much talked about in India about not only promoting participation but also improving the controlling function held by the lower levels of the political systems making more ordinary people influence and involve in the political process thus strengthening the state's capacity for implementation remained only on paper with little or no effect. Thus, in this case too the health staff at the cutting edge level in all terms were marginalized.

\section{Introducing HISP}

It is in this context Health Information Systems Programme (HISP), a global initiative was introduced initially as a pilot study in Kuppam, a small geographical area consisting of 9 PHCs spread over 5 mandals(administrative revenue divisions) in Chittoor district in the state of Andhra Pradesh, India.

As the main objective of our project is to strengthen information practices within the Primary Health Care (PHC) sector with the larger aim to improve processes concerning health care delivery for the rural community initial months were spent in trying to understand the structure and functioning of the PHCs along with the complex and multi-level flows of health information from the rural community to the department in state head quarters through the intermediary layers of the PHCs and district offices.

The district Health Information System(DHIS) that has been developed by HISP includes software, methodologies for health information management, training programmes, health data standards and a data dictionary.

Based on the discussions with the grass root level health functionaries and the officials at various levels efforts were made to set up the necessary infrastructure by placing computers in all the 9 PHCs of Kuppam constituency for initiating change through DHIS (District Health Information System),an open source software for improving their routine health information processes since more than $60 \%$ of the time of health workers is spent on health information transactions (collection of data, collation of data in various forms and registers, and the transmission of data to upper levels of district and state). It was also considered that improving these processes will then directly have an impact on work, and the health staff can focus more time on providing care to the community as compared to filling up endless forms that ultimately are marginal to their work of providing health care to the community. So keeping in view the above perspective strategies were devised to provide training to the health workers and other staff in the PHC and at the District Medical and Health Office(DM\&HO) with a view towards computerizing the 9 PHCs within Kuppam constituency and at the same time building capacity among health workers and other staff to work independently on the DHIS software, dealing with health information, and thus enabling a culture of "use of information. 


\subsection{A Brief Note About the Training Strategies}

Two main types of strategies were identified for the training and they were 'On-site' and 'Off-site'.

\section{On-site Training}

In the above type of training a trainer was based in each of the PHCs selected for piloting of the project from morning till evening for a period of six months providing training to the health staff.

The trainers provided training beginning from explaining concepts of HIS, basics of computer to making them familiar with working on MS word, MS Excel and teaching of DHIS - customised software on HISP.

Initially both the trainers and the health staff had many problems on both sides teaching as well as learning.

On teaching side the trainers faced the initial problem of gaining domain knowledge(health), explaining the concepts of HIS, advantages of HIS, familiarity of handling computer, time devoted by the staff in learning the concepts, and the software.

On learning side the heath staff faced the major problem of time and work load. Also in three of the PHCs among the nine in Kuppam the PHCs did not have the facility of keeping the computer(problem of infrastructure). As a result, the staff from the above three PHCs have to visit their neighbouring PHCs which in most cases were quite far from their own PHCs for getting training on DHIS software(problem of distances and remoteness). More over as there was only one computer there will be many people to be trained and in most of the cases many of the health staff will not get a chance to work practically on computer(shortage of resources).

\section{Off-site Training}

Once in a month a three day intensive training programme was organised in a computer training institute where HISP had entered into partnership for conducting training programmes for health staff.

In the above programmes the training was organised in such a way that some time is allocated for teaching in class about explaining the HIS concepts, various components about the DHIS software, importance of maintaining regular practice of doing data entry, importance of maintaining data quality, data analysis and how data can be converted into information and then how it can be used for making crucial decisions for instance, about relevant drug requirements, identifying reasons for epidemics or common outbreak of diseases in their wards or regions. Also during ITPs(Intensive Training Programmes) the health staff were trained to self monitor their work, the work of their sub centres and also make comparisons between the Sub centres in their PHC and also between different PHCs pertaining to any one or a cluster of performance indicators.

At the end of the ITP a feed back session was organised where the participants express their opinion about the training methodology, training content and their requirements for further training.

In spite of the hectic schedule and pressures from the higher authorities to achieve the irrational targets set on performance indicators majority of the health workers showed 
an interest in learning computers and the DHIS software and within seven months of time since the project was initiated most of the health workers quickly grasped the knowledge of using computers and DHIS software for doing data entry and doing simple analysis to monitor both individual and as well institutional performance in relation to the targets and indicators set by the higher authorities.

Many health staff in all PHCs started to use the application by regularly entering and updating the routine health monthly data in to the two forms of DHIS designed by the programmers in consultation with the users of the technology. The grass root health workers including the other health staff and the medical officer based at the PHC soon realised the benefits of using the application which not only reduced their manual work, duplication of collecting and collating data but helped them to analyse both individual and institutional performance both across time, indicators and institutions.

At the end of the eighth month after the project was initiated it was evaluated by the higher officials and once the authorities at the district and at the state were convinced of the use of computers, use of DHIS software and the quick technical skills gained by the health staff orders were issued from Commissioner of Family Welfare Department which mainly controls all the activities of the PHCs in the state for making transition to using reports generated from DHIS software in the place of using manual reports.

\section{Challenges}

While getting a Government Order from Commissioner of Family Welfare Department (CFWD) to institutionalise HISP was a great achievement for the implementation team but soon the team members realised that they have to face many problems in trying to stabilise the project due to non participation of officials at various levels. In the case of institutionalisation of HISP it was expected that all health staff including the health workers based at the cutting edge level concerned with HMIS will use the above application by doing data entry, analyse the data, generate reports and make use of the information using the DHIS software but in actual practice the senior staff within PHCs and authorities based at the District Medical and Health Office did not participate in the use of application mainly in generating of reports thus putting the whole process of implementation of HIS at stake. The problem was that the officials at DM \& HO refused to learn the computers and as well the DHIS software giving vague excuses such as work pressure, non availability of time and even expressing scepticism about the application itself thus insisting from the health staff from PHCs to submit manual reports along with a copy of reports generated from the DHIS application.

Both the health workers and other staff at the PHC felt that by submitting both the manual reports as well as the reports generated from computer using the DHIS application as demanded by the authorities at the district level that they are made to work in parallel on both the systems rather than on one as promised by the implementation team and the officials at the district level thus increasing their burden of work. This resulted in health workers losing interest in using the above application. 
Another challenge that posed serious threat to the sustainability to the project itself was again the generation of reports by health workers using DHIS. Little did the health staff realised at that time that generating reports using DHIS software would bring to the fore the stark realities of manipulation of data in the PHCs that has been going on for years. In Andhra Pradesh the health sector has selected 16 performance indicators such as Sterlisations, immunisations, institutional deliveries, number of Eligible Couples being motivated for undergoing button hole tubectomy(BHT), number of pregnant women registered for ANC before twelve weeks etc are some of the important performance indicators against which the PHCs were given targets to accomplish which again filters down to the field staff. Without taking into consideration the ground realities such as size, type and composition of the population and other duties of the health workers the policy makers based at the state head quarters fix the irrational targets for the health workers based at the field to achieve them. In one case a health worker responsible for a Population Project(PP)unit in Kuppam constituency was asked to complete 11 cases of sterlisations per month. To achieve that target the PP Unit should have a population of 11000 population but the above unit had only 5000 population and while many times the health worker has highlighted the mismatch between the size of the population and the targets fixed on sterlisations and pleaded with the authorities to fix the rational targets it has not been taken into consideration and in turn the authorities kept pressurising the health worker to achieve the targets. These targets have been unrealistic in the sense that in majority of the cases they are beyond the stage of being fulfilled. Instead of questioning the irrationality of targets and demanding for rational targets from higher authorities the practice for years has been to manipulate the data particularly at the time of collating and reporting in the formats at the PHC level. This is done by the health workers, health supervisors and medical officers to escape the wrath of the higher authorities and to get good grading for their institutions. The participation of the users in the process of implementation of HIS remained fine till the point of data entry but little did they realised that manipulation of data cannot be done while generating reports.(The DHIS generated monthly progress reports of PHC,PPU,UFWC,UHC, Family Welfare Performance report, Family welfare performance institution-wise report and MTP[Medically Terminated Pregnancy report] and many more required by the DM\&HOs office).After completing data entry in DHIS and when reports were generated both the supervisor and medical officer were shocked that figures shown in the reports were far below the targets given by the authorities. Since the reports were printed using the application they could not change the figures. And there was no option to generate empty formats of the reports in the DHIS software and so the opportunity of filling the figures manually was not present. Soon the staff at the PHC realised that computerised reports designed with their participation did not allow them to manipulate the data which they had been used to.

Within short span of time the numbers of PHCs generating reports using DHIS software dwindled. Initially they gave excuses but soon they came with the request of developing the option in the software to manipulate the figurers. Since improving quality of data is one of our main objective the team discussed the above issue with 
the medical officers in getting their targets fixed rationally using the information about their geographical area, population and other criteria but it is of no use as the concept of decentralised information which is much spoken is still far behind in practice.

While many of the staff in the PHC justify the manipulation of data to the unreasonable targets that are fixed by the policy makers without taking into consideration the actual situation in the field that exists others mutely accept the fact and to save themselves from the wrath of being punished and humiliated do not want to use the systems. Most of them looked for loopholes in the systems where the figures can be changed automatically while the reports are printed to be submitted to the district officials but it was futile. The state proclaims several things but rarely reaches down to the base level of society[4] .

At that time, implementation team continuously motivated the users to complete the data entry in the DHIS software and generate the reports using the above application and then using the information demand for rational targets to be fixed from the higher authorities. But the users were in dilemma. Since the users at the higher level that is, the policy makers based at the state head quarters did not participate in the implementation of HIS the staff at the field level reverted back to manual systems of collecting, collating and making manual reports. Thus implementing change is more difficult and contrary to the notion that in developing countries anything which comes free particularly technology will be easily accepted and can be implemented is a fallacy. Thus non participation of officials at various levels and to some extent by the users based ay the grass root level has put all the efforts of implementation team and as well the project itself at stake.

\section{Analysis}

Though much is spoken about decentralization in India still everything remains centralized and even the basic right to information which is necessary for citizens to participate in governance, especially at local levels is denied by those in authority as according to them information is power. The bureaucracy in India still keeps up the colonial culture of secrecy, distance and mystification [5].

According to Ostrom's study (1990) in order to bring about sustainable reforms, a process of change must take place in the local community. Central government can facilitate such a development in several ways, but it cannot accomplish the process by itself and this can only be done by local actors on the ground. To a great extent it is a matter of mobilizing and empowering the local community. Hence, local democratisation is an essential part of the 'reform package'.

But generally what happens is that due to the basic weakness that prevails in centralisation decision-making is inflexible to varying local demands and conditions. In addition, central actors are severely restrained when it comes to monitoring the actual implementation of programmes out in the field. Involving local actors with an independent responsibility for accomplishing certain activities may mitigate these drawbacks. This is a fundamental argument for decentralisation. The problem, however, is that existing local decision-makers often have their own policy agenda and are therefore likely to divert programmes for their own ends, to the detriment of 
the community at large [6]. To counteract this tendency, it is necessary to inspire the stakeholders - the intended beneficiaries - to become involved in the process .

So, in a country like India where hierarchy and bureaucratic structures are strong it is important first to bring about a change in the organization and hierarchical structures. During the process of any implementation it is imperative to give importance to both technical and organizational issues since organisational issues play a crucial role in making any implementation a success.

\section{References}

1. Bhattacharyya, D.: Limits of Legal Radicalism: Land-Reforms and the Left Front in West Bengal. Calcutta Historical Journal 16(1), 57-100 (1994)

2. Ostrom, E.: Governing the Commons: The Evolution of Institutions for Collective Action. Cambridge University Press, Cambridge (1990)

3. Hadenius, A.: Decentralisation and Democratic Governance.Experiences from India,Bolivia and South Africa. ed. Almqvist \& Wiksell, International, Stockholm (2003)

4. Mander, H., Joshi, A.S. The Movement for Right to Information in India: People's Power for the Control of Corruption, Commonwealth Human Rights Initiative (reprinted 2001) (1999)

5. Manor, J.: The Political Economy of Decentralisation, The World Bank, Washington D.C (1997) 